\title{
Rats' responses to a moving object related to food or water: A behavior-systems analysis
}

\author{
WILLIAM TIMBERLAKE \\ Indiana University, Bloomington, Indiana
}

\begin{abstract}
The present experiments compared rats' responses to a moving object (a rolling ball bearing) related to either food or water under both Pavlovian and operant contingencies. In Experiment 1 , food-restricted rats contacted food-related bearings more frequently and with more complex response patterns than water-restricted rats contacted water-related bearings. Foodrelated contacts occurred with shorter latency, longer average duration, and increased likelihood of dig, carry, and chew. Experiment 2 revealed that once contact with the bearing had been established, its form persisted despite changes in the type of reward and restriction. In Experiment 3, rats that were simultaneously food and water restricted learned to discriminate between painted and unpainted bearings related to food versus no food, water versus no water, and food versus water. Again, food-related bearings produced more complex, although not more frequent, interactions than did water-related bearings. In none of the experiments did rats lick the ball bearing related to water. The results supported a behavior-system approach, but not the stimulus-substitution or arbitrary-operant accounts of conditioned-response topography.
\end{abstract}

Rats are predatory animals that may respond to small moving prey with a response sequence of digging, chasing, seizing, and various killing and/or food-handling behaviors (Karli, 1956; Timberlake, Note 1). Timberlake, Wahl, and King (1982) engaged most of these behaviors by presenting a rolling ball bearing related to the delivery of a food pellet. After repeated Pavlovian pairings or operant contact contingencies, rats typically seized the bearing near the entrance hole, stuffed it into the mouth, and ran to the end of the chamber near the food tray, where (unless interrupted by the delivery of food) they handled, chewed, dropped, and retrieved the bearing for lengthy periods before finally releasing it. Related results were reported by Boakes, Poli, Lockwood, and Goodall (1978; see also Boakes \& Jeffery, 1979), who used an operant contingency that required rats to carry a ball bearing to a chute and deposit it in order to obtain food.

Timberlake (1983, in press) and Timberlake et al. (1982) interpreted these results as supporting the importance of preorganized stimulus sensitivities and response patterns in determining the nature of learned performance. The basis for such appetitive structure presumably evolved within functional sys-

This research was supported in part by National Science Foundation Grant BS 82134556 and Biomedical Research Support Grant S07-RR07031 from the National Institutes of Health. I particularly thank Sebastiao Feliz for his assistance in performing and analyzing the results of these experiments. Thanks also go to Gary Lucas and Don Gawley for comments. My mailing address is: Department of Psychology, Indiana University, Bloomington, Indiana 47405. tems of behavior because of increased efficiency in obtaining important commodities (terminal events), such as food, water, and shelter. Within the behaviorsystem view, ready conditioning of preorganized response patterns should occur when the appropriate system is potentiated and the stimuli predicting the terminal event resemble those cues ordinarily eliciting and controlling appetitive behavior in more natural settings.

The resemblance between predictive stimuli in the laboratory and naturally occurring cues is based on both physical and temporal characteristics. Thus, presenting a rat with a moving object (a rolling ball bearing) predicting the imminent delivery of food should increase predatory behaviors directed to the bearing, whereas presenting a moving object temporally separate from food should be much less effective (Timberlake et al., 1982). On the other hand, presenting another rat as a predictor of food should increase social, rather than predatory, behaviors in this social-feeding species (Timberlake, in press; Timberlake \& Grant, 1975), whereas the pairing of a conspecific with food should decrease social behavior in predominately nonsocial feeders, such as hamsters (Timberlake, in press).

Such predictions and confirming results are at variance with the traditional stimulus-substitution view of Pavlovian conditioning (Mackintosh, 1974; Pavlov, 1927) and with its extension to account for the form of operant responses (Hull, 1977; Moore, 1973). In this view, important elements of the topography of Pavlovian or operant conditioned responses should be highly similar to the unconditioned behavior in the presence of reward. Thus, in the cases 
above, the animals should have reacted to the predictive stimulus with components of the ingestive behavior directed to the food pellets, instead of with components of other natural appetitive behaviors.

The present experiments further contrasted the predictions of the stimulus-substitution and behaviorsystem accounts of topography by examining the behavior of rats in response to rolling ball bearings related to either food or water. Although the traditional stimulus-substitution hypothesis is not adequate to handle the conditioning of the complex social and predatory behaviors outlined above, it has generally been given passing marks in accounting for differences in responses related to food and water reward. Davey and Cleland (1982), Hull (1977), and Jenkins and Moore (1973), among others, have emphasized the resemblance between the topography of the learned response and the unconditioned ingestive response in the case of food and water reward. In this view, a ball bearing related to food should be picked up in the animal's mouth, chewed, and perhaps swallowed. A bearing predicting water should be licked and perhaps sniffed.

In contrast, because of the ball bearing's movement and temporal proximity to reward, the behaviorsystem view predicts that rats should increase predatory behaviors such a digging, seizing, carrying, chewing, and retrieving directed to a bearing predicting food. Prediction of behavior directed to a bearing followed by water is more difficult because of overlap between the food and water systems. Rats, like many other rodents, secure some fluids from live prey, so there may be similarity in evolved stimulus sensitivities in the food and water system. Second, because food and water deprivation are not independent states at the level of physiology or behavior (e.g., Bolles, 1975, pp. 165-166), restricting either food or water is likely to potentiate both systems. However, since most water intake in rats appears to be from surface water and vegetable matter rather than from prey, and since the feeding system should be less strongly potentiated by water restriction than by food restriction, rats should interact less often and less elaborately with bearings that predict water than with those that predict food. Furthermore, little licking of a bearing predicting water would be expected, because a bearing would provide minimally appropriate form, taste, and moisture cues, and because drinking probably would be inhibited initially by the engagement of a predatory capture sequence.

Experiment 1 compared the reactions of different groups of rats to ball bearings related to food or water under both Pavlovian and operant contingencies. Experiment 2 reversed the reward and restriction conditions for the two groups used in Experiment 1 in order to determine to what extent behavior conditioned in the presence of one reward persisted in the presence of a different reward. Experiment 3 used operant-discrimination procedures to compare reactions of the same animals to painted and unpainted ball bearings predicting food or water versus nothing and food versus water. Experiment 3 also removed the differential deprivation conditions that existed for the first two experiments, thereby illuminating the relative contribution of drive level and reward in producing learned behavior.

\section{EXPERIMENT 1}

The purpose of Experiment 1 was to compare the reactions of different groups of animals to ball bearings related to food or water under both Pavlovian pairings and operant contingencies requiring simple contact of the bearing with the mouth, nose, or paw. The stimulus-substitution view predicts the emergence of mouthing of the ball bearing by the food group and licking of the ball bearing by the water group. The behavior-system view predicts the emergence of components of the natural predatory sequence for the food group, including gnawing of the bearing (Timberlake et al., 1982) and less complex and frequent interaction with the bearing for the water group (see Boakes et al., 1978, Davey \& Cleland, 1982 , and Hull, 1977). Both viewpoints predict that the topography of behavior under Pavlovian and operant procedures should be relatively similar. The behavior-system prediction is based on the common underlying appetitive structure and stimulus sensitivities. The stimulus-substitution prediction is based on the fact that Pavlovian pairings between the bearing and reward occur under both types of contingency (Hull, 1977).

An important problem in any study using different deprivation states is the possibility that differences in behavior may be due to differences in level rather than in type of motivation. This objection is less strong in comparing the effects of food and water restriction, because the levels of the two drives are linked; nonetheless, it must be considered. A reasonable approach to the problem is to vary the amount of deprivation across a wide range to determine if different behavioral results are produced by different levels of the same drive. Boakes et al. (1978), Davey and Cleland (1982), and Hull (1977) all manipulated the drive variable and found little indication that the primary behavioral differences between food and water reward could be reproduced by changes in the level of either drive. In the present experiment, we dealt with the problem by equating body weight in food-restricted and water-restricted groups, since body weight has been strongly implicated in drive effects (e.g., Bolles, 1965). Furthermore, we inspected baseline responding to the ball bearing in the absence 
of reward to determine if there were any driveproduced differences in initial reactivity prior to learning. There were not.

Experiment 1 was divided into three major conditioning procedures. The initial procedure consisted of modified Pavlovian delay pairings between the presentation of the ball bearing and the delivery of food; in these pairings, reward did not come until the bearing had exited the chamber. Thus, any interaction with the bearing delayed the delivery of reward. In the second procedure, the rats were required to contact the bearing in order to receive reward. In the third procedure they were briefly extinguished to disrupt any goal-oriented behaviors acquired in the Pavlovian procedure and then again conditioned to contact the bearing. This set of conditions was preceded by a baseline condition and was followed by extinction.

\section{Method}

Subjects. The subjects were 12 Sprague-Dawley female albino rats, approximately 110 days of age at the beginning of the experiment. The rats were housed individually under a 12-h:12-h light:dark cycle; they were run approximately halfway through the light period. The food-deprived subjects were maintained at $85 \%$ of their free-feeding weights by being fed a small amount of food an average of $45 \mathrm{~min}$ after they were run. The water-deprived subjects were provided 15 -min access to water, beginning an average of $\mathbf{4 5}$ min after they were run.

Apparatus. The apparatus was a rectangular sheet-metal box, $61 \times 30 \times 30 \mathrm{~cm}$, with a Plexiglas top and front. Operation of a modified BRS feeder dispensed 1.6- $\mathrm{cm}(5 / 8-\mathrm{in}$.) ball bearings through a hole into an angle-iron channel that ran the length of the apparatus to an exit hole. The $V$-shaped channel and floor of the apparatus were slanted approximately $6 \mathrm{deg}$ from the entrance to the exit holes, and the floor was slanted toward the channel such that a bearing dropped anywhere in the apparatus rolled toward the channel and out of the apparatus. The channel and holes were $9 \mathrm{~cm}$ from the front wall of the apparatus. A ball bearing entered the apparatus approximately $2 \mathrm{sec}$ after operation of the bearing dispenser and, if undisturbed, left the chamber $3.1 \mathrm{sec}$ later; thus, the time elapsed from operation of the dispenser to exit of the ball bearing was $5.1 \mathrm{sec}$.

The food and water receptacles were $2.0 \times 5.0 \times 1.8 \mathrm{~cm}$ deep and were located in a two-receptacle frame $6.4 \mathrm{~cm}$ from the floor of the chamber and $12 \mathrm{~cm}$ to the right of the exit hole. The relative position of the food and water receptacles was counterbalanced across subjects. A Skinner valve and flow restrictor delivered water $(.10 \mathrm{ml})$ into a small brass cup in the bottom of the receptacle. A Waltke feeder (Waltke Scientific Enterprises, Psychology Department, Indiana University) delivered the food (.045-mg Noyes pellets). The observer sat $1 \mathrm{~m}$ from the front wall of the apparatus. Data-recording and control equipment were located in the same room.

Procedure. The animals were divided into groups of six each, matched on their initial body weights. The two groups received different rewards (food or water) and different deprivation schedules, but otherwise were treated identically. Each animal received the following six conditions: (1) pretraining - 1 day of exposure to the room, 1 day of 10-min exposure to the chamber with five pellets or $1 \mathrm{cc}$ of water present in the appropriate receptacle, and 1-2 days of training to approach and eat from the food or water receptacle to a criterion of three out of four approsches to the operation of the food (or water) dispenser; (2) baseline-4 days on which the ball bearing alone was presented on a variable-time (VT) 45-sec schedule; (3) Pavlovian conditioning - 16 days on which the exit of the ball bearing was followed by food or water; (4) operant conditioning -12 days on which food or water followed exit of the ball bearing if the rat had previously contacted the bearing; (5) brief extinction- 6 days of presentation of the ball bearing alone; (6) operant reconditioning-12 days of exposure to the same operant contingency as above; and (7) extinction-14 days of presentation of the ball bearing alone.

Under all procedures except pretraining, each subject received 12 presentations of the ball bearing on a VT $45-\mathrm{sec}$ schedule. Between subjects, the ball bearings and the apparatus were washed with a weak organic-acid detergent mixture to remove any animal and reward odors that remained. The order of running was changed each day, and the animals received their daily ration of food or water an average of $\mathbf{4 5}$ min after they had run.

On each trial, at least one observer coded the animal's behavior into the categories of dig (move the forepaws at the entrance hole just prior to the entrance of the bearing), carry (locomote with the bearing in the mouth), sniff (contact the bearing with the nose, usually obviously accompanied by rhythmic whisker and nose movements), lick (contact the bearing rhythmically with the tongue), chew (contact the bearing rhythmically with the teeth while holding it in the front paws), and contact (any of the above plus paw contacts), and noted additional comments in the margin of the coding sheet. The categories are more fully defined in Timberlake et al. (1982). The same observer coded behavior for all the present experiments. The observer was trained as outlined in Timberlake et al. (1982), and the correlation of coding with the training observer was .96. The observer used a set of buttons connected to cumulative timers to record duration and latency of contact on each trial. When there were clear predictions of the direction of effects, one-tailed tests were used in analyzing the data.

\section{Results}

Figure 1 shows the mean percentage of trials with a contact by 2-day blocks as a function of groups and conditions. Figures 2 and 3 show average duration and latency of contact, and the average percent contact trials with a carry or chew at asymptote for each group and condition. Asymptotic responding was based on average three-trial medians for each group.

Pavlovian procedure. Figure 1a shows that the Pavlovian procedure produced a modest increase in the percentage of trials with a contact, particularly for the food group. Five of the six animals in the food group increased their contacts, from baseline to asymptote $[t(5)=2.53, p<.05]$, but only two of the animals in the water group reliably increased contacts $[t(5)=1.16, p>.20]$. The remaining animals went to the water receptacle instead (goal tracking, Boakes, 1977). Because the two water animals that increased contact did so vigorously, the two groups overlapped in contacts at asymptote $[t(10)=1.49, .10>p>.05]$.

Figure 2 shows that, under the Pavlovian procedure, the average duration per contact increased significantly over baseline levels for the food animals $[t(5)=2.16, p<.05]$, but not for the water animals $[t(5)=1.19, p>.10]$. The average latency per contact decreased for both the food and water groups [ts(5) $=5.62$ and 2.32, ps $<.01$ and .05$]$. Also, the food group contacted significantly longer and with shorter latency than did the water group at asymptote [ts(10) 


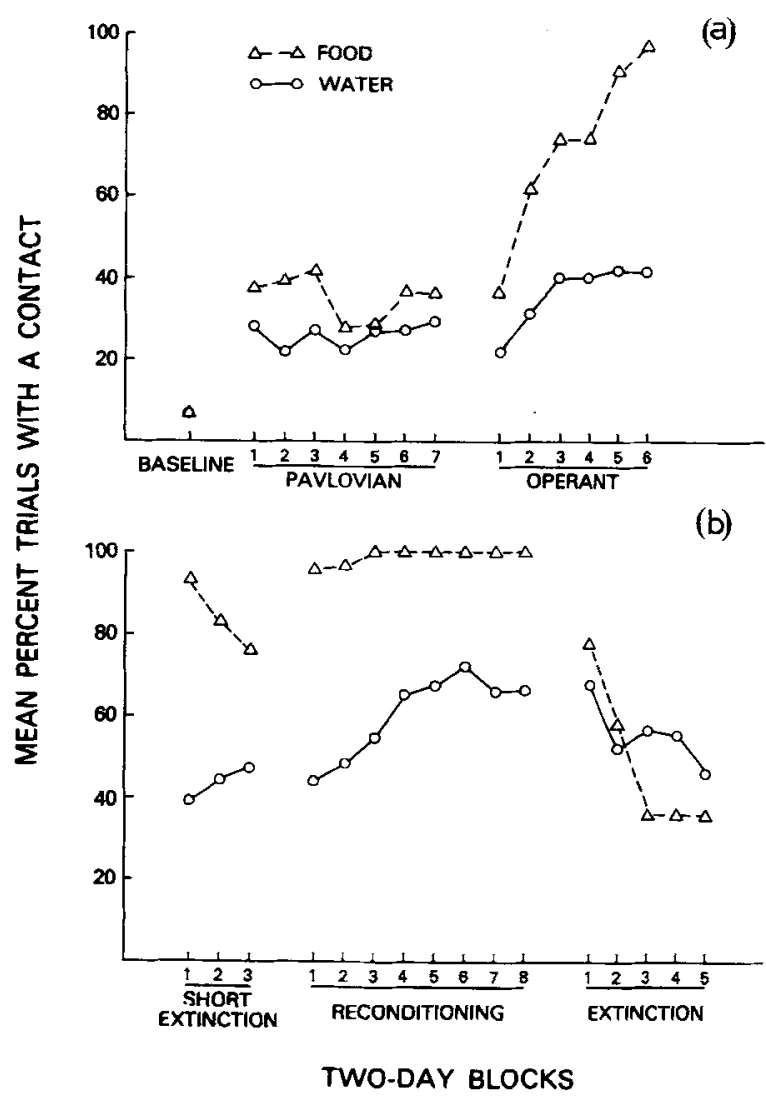

Figure 1. (a) Average percent trials with a contact by food and water groups during baseline (medians of Days 2-4), Pavlovian conditioning, and operant conditloning (all 2-day averages) in Experiment 1. (b) Percent trials with a contact by food and water groups during brief extinction, reconditioning, and extinction (all 2-day averages) in Experiment 1.

$=6.03$ and 2.87 , ps $<.011$. Figure 3 shows that the likelihood of carry and chew per contact trial increased over baseline only for the food group [ts(5) $=2.49$ and 2.49 , ps $<.05$ ], and at asymptote, the food group exceeded the water group on both measures $[\operatorname{ts}(10)=3.11$ and 3.01 , ps $<.01]$. Last, the likelihood of nose and dig increased for three animals in the food group, but for only one animal in the water group.

Operant procedure. Figure $1 \mathrm{~A}$ shows that the operant procedure of requiring contact with the bearing for delivery of food or water was successful in increasing contacts for both the food and the water groups [ts $(5)=6.29$ and 2.24 , ps $<.01$ and .05 ]. Figure 2 shows essentially the same duration and latency effects as those found under the Pavlovian procedure. The food group contacted with significantly shorter latency and longer duration than did water group [ts $(10)=3.00$ and 3.07 , ps $<.01]$. The only significant change from the Pavlovian to the operant conditions was an increase in the average duration of contact by the food group $[t(5)=4.75$, $\mathrm{p}<.011$.

Similar results are shown in Figure 3 for the occurrence of carry and chew. There was no significant change in either measure for either group as a result of the operant contingency (all ps $>.10$ ). The food group carried and chewed significantly more than did the water group $[\operatorname{ts}(10)=2.48$ and 2.13 , ps $<.051$. Furthermore, the likelihood of sniff increased from $0 \%$ during baseline to $56 \%$ of the contact trials for both the food and the water groups $[\operatorname{ts}(5)=3.17$ and 3.06 , ps $<.05$ ], but did not differ between the groups [t $(10)<1]$. The likelihood of dig increased only for the food group (from $0 \%$ to $23 \%$ of the contact trials) $[t(5)=3.21, p<.05]$, and the food group significantly exceeded the water group at asymptote $[t(10)=2.64, p<.05]$.

Reconditioning. Figure $1 \mathrm{~b}$ shows that the brief extinction increased the effectiveness of the operant contingency in producing contacts in the water group $[t(5)=2.29, p<.05]$. Presumably, this resulted from the extinction of competing goal-oriented behaviors

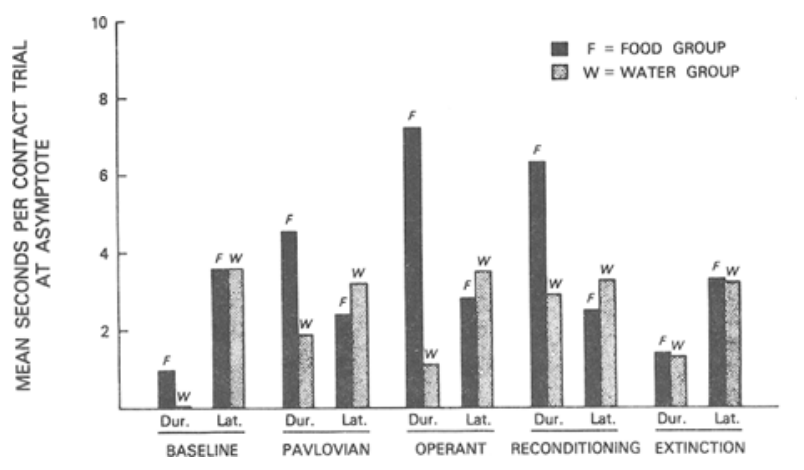

Figure 2. Average duration and latency of contact at asymptote (3-day medians) during baseline, Pavlovian conditioning, operant conditioning, reconditioning, and extinction (Experiment 1).

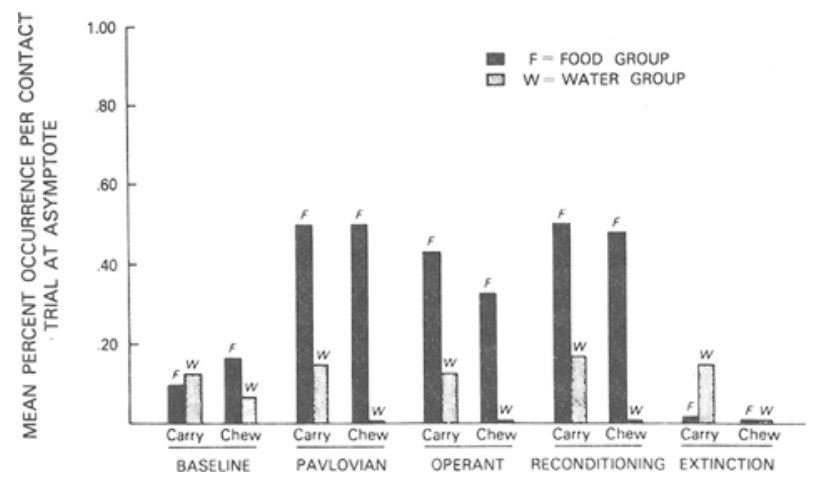

Figure 3. Average percent of contact trials with a carry or chew at asymptote (3-day medians) daring basellae, Pavlovian conditoning, operant conditioning, reconditioning, and extinction (Experiment 1). 
that had developed in previous training. However, the food group still contacted the bearing significantly more often than did the water group $[\mathrm{t}(10)=$ $2.65, p<.01$ ]. Figure 2 reveals that the average duration of contact increased over the level achieved in the previous operant training for the water group but not for the food group $[\operatorname{ts}(5)=1.94$ and $<1$, ps $<$ 1.0 and $>.10]$, and the average latency per contact decreased significantly for the food group but not for the water group $[\operatorname{ts}(5)=2.71$ and $\langle 1$, ps $=.05$ and $>$ $.10]$. Under reconditioning, the food group contacted significantly longer and with shorter duration than did the water geroup $[\operatorname{ts}(10)=2.76$ and 2.03 , ps $<.01$ and .05]. Figure 3 reveals that the likelihood of carry and chew remained stable between the two operant procedures $[\operatorname{ts}(5)<1, p>.10]$, but that the food group still chewed and carried during a contact more frequently than did the water group $[\operatorname{ts}(10)=1.82$ and 2.16 , ps $<.05$ ]. The likelihood of sniff increased significantly over the previous condition for the water group but not for the food group $[\operatorname{ts}(5)=3.15$ and $<1$, ps $<.05$ and $>.10$ ].

Extinction. Under final extinction, all measures decreased for all groups (all at least $p<.05$ ), and the food and water groups did not differ from each other [all ts $(10)<1$, ps $>.10$ ].

\section{Discussion}

Both Pavlovian and operant procedures increased contact with the ball bearing. The operant procedure appeared to be considerably more effective in producing contact, but the topography of the contact was very similar in the two cases. There was no change in the likelihood of carry and chew and only relatively small differences in duration and latency of contact across the Pavlovian and operant contingencies. The results also showed consistent differences across both procedures in the behavior of the food and water groups. The food animals tended to contact the bearing more frequently, and when they contacted, they did so with shorter latency, for longer duration, and with more digging, carrying, and chewing of the ball bearing.

Considering previously reported levels of contact with the bearing under Pavlovian procedures (Timberlake et al., 1982), the present results were disappointing. However, on the basis of unpublished results from our laboratory, a likely explanation is that the present pretraining with the unconditioned stimulus (US) was too extensive, which produced goal-oriented behavior (goal tracking, Boakes, 1977) that interfered with approach to the ball bearing. In that application of an operant contact requirement had only a slight effect on frequency of contact until the response pattern had been exposed to a brief extinction phase preceding reconditioning, it appeared that this goal-oriented behavior was more strongly conditioned in the water group than in the food group. Such a differential susceptibility to the conditioning of goal-oriented behavior by the water group is compatible with the results obtained by Reberg, Innis, Mann, and Eizenga (1978) with pigeons and with those obtained by Reberg, Mann, and Innis (1977) with rats. In both experiments, subjects given periodic water reward remained much closer to the hopper than did groups given periodic food reward. Such an outcome would make sense from an evolutionary view if water were a commodity that tended to be more widely dispersed and quickly replenished than food (e.g., water may rapidly fill in a depression from which it has been drunk).

On the whole, these results support the behaviorsystem approach rather than the stimulus-substitution hypothesis. First, in the case of neither the food nor the water rats were behaviors that were directed at the ball bearing very similar to those directed to the reward (US). The food rats showed a much more complex topography of responding to the ball bearing than to the food pellets: They dug, seized, carried, and chewed (gnawed) the bearings, behaviors that never occurred in response to the pellets. In the case of the water rats, although their dominant response to the water US was licking, they were never observed to lick the ball bearing. In fact, the two water rats that contacted the bearing most frequently showed the same contact characteristics as the food rats. As a group, the water rats did increase the amount of sniffing of the ball bearing, but this effect was matched by the food group until the reconditioning procedure.

Second, the topography of responding to the bearing by the food rats was quite compatible with the predatory behaviors directed by rats at insects (Karli, 1956; Timberlake, Note 1). The results strongly suggest that presentation of a small moving stimulus preceding food makes these behaviors readily available for conditioning under either Pavlovian or operant contingencies. The behaviorsystem approach also explains why the majority of the water rats did not show extensive and complex interactions with the bearing, since these behaviors would be more appropriate for a food object. More difficult to explain is the behavior of the two water rats that showed behavior virtually indistinguishable from that of the food animals. As indicated in the introduction, this may have been because rats obtain some fluids from prey or because of the linkage of food- and water-drive systems. This overlap in behavior is further addressed in Experiment 3 and in the General Discussion.

\section{EXPERIMENT 2}

The intent of Experiment 2 was to explore briefly the effects of prior training with one reward on the pattern of behavior directed to the ball bearing when 
it was related to the other reward. Boakes et al. (1978) reported mixed data: Food-rewarded animals that were switched to a water reward persisted in their previous levels of responding; however, waterrewarded animals that were switched to a food reward gradually increased the time between obtaining and finally depositing the bearing. Since all animals in the Boakes et al. (1978) experiment were on a common deprivation schedule on which both food and water were restricted, it may be that the food animals persisted in their previous levels of responding because of their continued levels of food deprivation.

Hull (1977) reversed the deprivation levels and the reward for barpressing and got a very rapid increase in long-duration barpresses for the new food group and a much slower decrease in long-duration barpresses for the new water group. The present experiment also reversed the commodity restricted at the same time that it switched the reward and, in addition, waited 8 days to allow the animals to adjust to their new restriction condition. Despite these precautions, the results indicated a very strong persistence of previously conditioned behavior under the new combination of restriction and reward.

\section{Method}

Subjects, Apparatus, and Procedure. The subjects from Experiment 1 were reversed in their deprivation conditions, such that the previously food-restricted rats were now deprived by limits on their water intake and the previously water-restricted rats were now deprived by limits on their food intake. Following 8 days of adjustment to this schedule, the rats were run first for 4 days of baseline, during which the ball bearing was presented alone, and then for 12 days under an operant contingency requiring contact with the bearing for a reward appropriate to the rats' new deprivation schedule. The contingency condition was followed by 14 days of extinction.

\section{Results and Discussion}

Figure 4 shows that switching deprivation regimes and rewards had little effect on behavior. The new

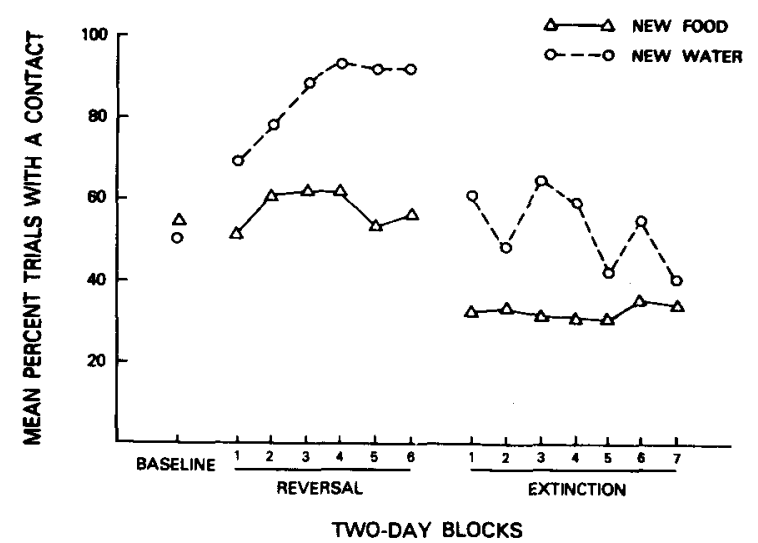

Figure 4. Average percent trials with a contact by new food (old water) and new water (old food) groups during baseline (3-day medians), operant conditioning, and extinction (2-day averages) in Experiment 2. water group (old food group) continued to contact the bearing during conditioning more frequently than did the new food group (old water group) $[t(10)=$ $2.26, \mathrm{p}<.05$ ], and there was little indication that this difference decreased over conditioning trials. Previous differences between the groups in terms of duration and latency of contact, and percent contact trials with a carry and chew, also tended to persist. The new water group still contacted for longer duration and with shorter latencies than did the new food group $[\operatorname{ts}(10)=1.96$ and $2.40, .10>p>.05$ and $p<$ $.05]$. The new water group also continued to carry significantly more often than did the new food group $[t(10)=2.08, p<.05]$, although the difference in the likelihood of chew disappeared $[t(10)>1]$, due in great part to a general reduction in chew.

In short, the two groups maintained the majority of their previously conditioned behaviors despite reversals in deprivation regime and type of reward. The differences were generally less marked, but showed no tendency to reverse. These findings are partly consistent with those of Boakes et al. (1978) and Hull (1977), who also found that animals trained under food reward persised in their length of contact under water reward. However, in both these cases the new food-rewarded animals significantly increased their length of contact with the manipulandum. The failure of the water-trained animals in this study to change under food reward may have been due to differences in the instrumental requirement or to the magazine-directed behaviors that developed. The present results apparently add another circumstance in which characteristics of instrumental responding persist despite changes in the consequences and the drive state (see Adams \& Dickinson, 1982).

\section{EXPERIMENT 3}

The results of Experiment 1 suggested important differences between food- and water-restricted animals in their interaction with ball bearings predicting the appropriate reward. On the basis of several arguments, it seemed unlikely that these differences in behavior were due to differences in levels of food or water motivation; however, it was not clear to what extent these differences were due to the potentiating and eliciting effects of the dominant drive state or to the type of reward. The present experiment attempted to explore further the relative importance of the drive state and the reward in determining reactions to the bearing by imposing a common multiple-drive state and varying the reward.

Subjects with experimenter-restricted intake of both food and water were exposed to two discrimination procedures in which different rewards were associated with painted or unpainted ball bearings. In Discrimination 1, the subjects received either food or water following contact with one type of ball bear- 
ing, whereas contact with the other type produced no reward. In Discrimination 2, all subjects received food following contact with one type of ball bearing and water following contact with the other. To the extent that behavior in response to the ball bearing is determined more by the drive state, we would expect similar behaviors to bearings regardless of type of reward or its presence or absence. To the extent that behavior is more influenced by the type of reward, we would expect different behaviors in response to bearings predicting food versus water and those predicting reward versus nonreward.

\section{Method}

Subjects. The subjects were 10 female Wistar albino rats, 90 days of age at the start of the experiment. Four of the rats were run initially with water reward, and 6 were run with food reward. Housing and treatment were the same as in the previous experiments, except that both food and water were restricted. Following each daily session, the animals were allowed 15-min access to water and enough food to maintain them between $80 \%$ and $85 \%$ of adlib. feeding weights.

Apparatus. The apparatus from the previous experiments was used. A second type of ball bearing was created by painting bearings so that all but the edge of each quarter of the surface of the sphere had white lacquer on it. Each quarter was separated from the others by a 5-mm unpainted band. When the bearing rolled, painted and unpainted surfaces alternated. Also, a second reward receptacle was again added to the end of the box. When not in use, it was covered with a plate. The position of the receptacle containing reward, or a particular type of reward, was counterbalanced across subjects.

Procedure. Handling, adaptation, and baseline procedures were the same as in Experiment 1. Food-tray training differed in that the animals were trained on both food and water reward; in no case did the total number of presentations of any reward exceed 16 , with at least 8 presentations being given while the animal was farther than $15 \mathrm{~cm}$ from the reward receptacle. During Discrimination 1 , the animals were run 16 trials a day for 16 days. On a random 8 of the 16 daily trials, one type of bearing was presented; the other type of bearing was presented on the remaining 8 trials. In the water group, water was presented following one type of bearing and nothing was presented following the other. In the food group, food was presented following one type of bearing and nothing was presented following the other. Discrimination 2 consisted of 12 days of a similar procedure, in which food was presented following one type of bearing and water was presented following the other. The relation between type of bearing and what it predicted was counterbalanced within and between conditions. Twelve days of extinction followed.

\section{Results}

Discrimination 1. Figure 5 shows a marked increase in the percentage of trials with a contact in Discrimination 1 for all reward conditions. An analysis of variance examining type of reward, presence of reward, and trials revealed a significant effect of trials $[F(7,120)=9.68, p<.01]$ and a small, but significant, effect of reward in producing more contacts than did no reward $[F(1,120)=5.53, p<.05]$. However, there was no effect of type of reward (food vs. water) $[\mathrm{F}(1,8)<1, \mathrm{p}>.10]$.

Figure 6 suggests that animals with food reward contacted for a longer average duration than did the

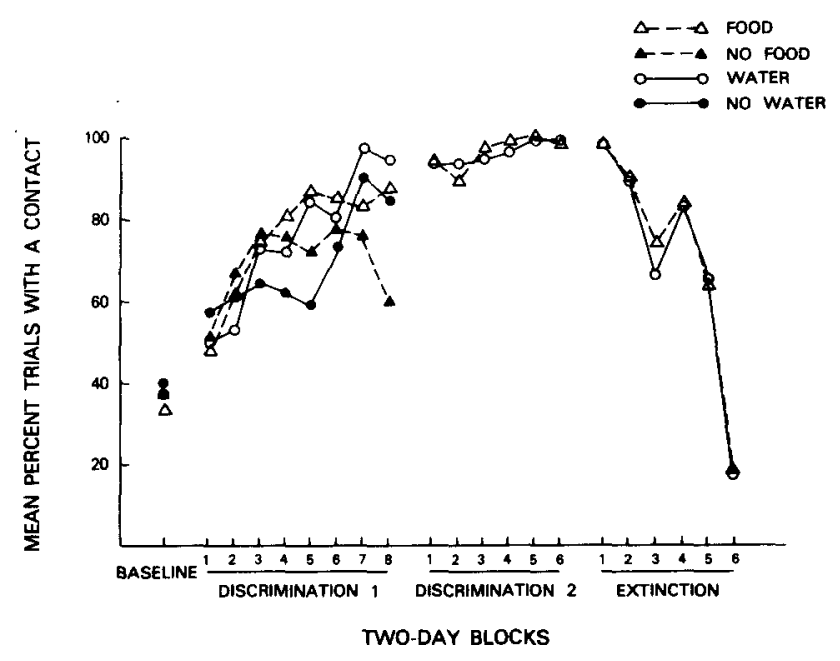

Figure 5. Average percent trials with a contact of bearings related to food, water, or nothing during baseline (3-day medians), Discrimination 1, Discrimination 2, and extinction (all 2-day avernges) In Experiment 3.

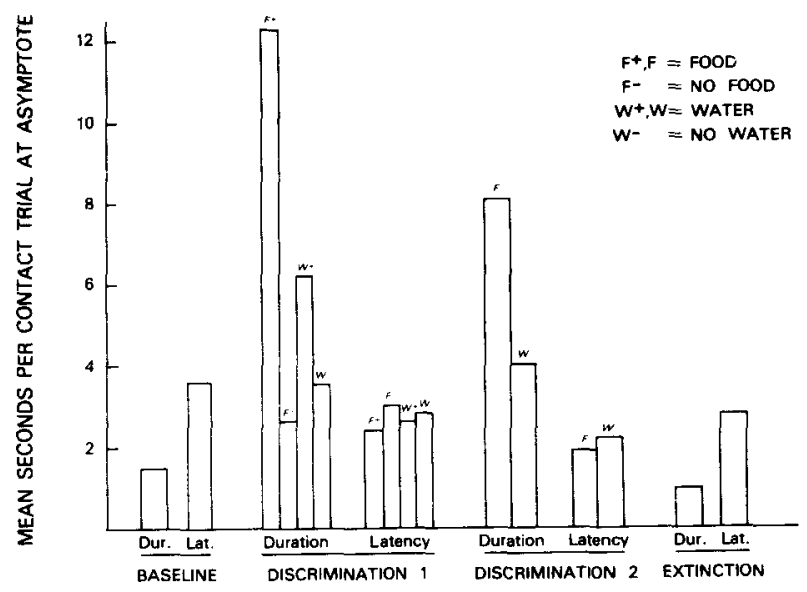

Figure 6. Average duration and latency of contact of bearings predicting food, water, or nothing (3-day medians) during baseline, Diserimination 1, Diserimination 2, and extinction (Expertment 3).

water animals. However, individual variance was high (in particular, one food animal showed an average duration of contact of $.6 \mathrm{sec}$ ), and the apparent difference was not significant. The animals did contact a bearing that predicted reward significantly longer than they did a bearing that predicted no reward $[F(1,8)=6.08, p<.05]$. Nine of 10 animals also contacted reward bearings with shorter average latency $[F(1,8)=4.50, .10>p>.05]$, and in all cases contact was with shorter latency than it had been in the baseline. Again, there was no effect of type of reward. 


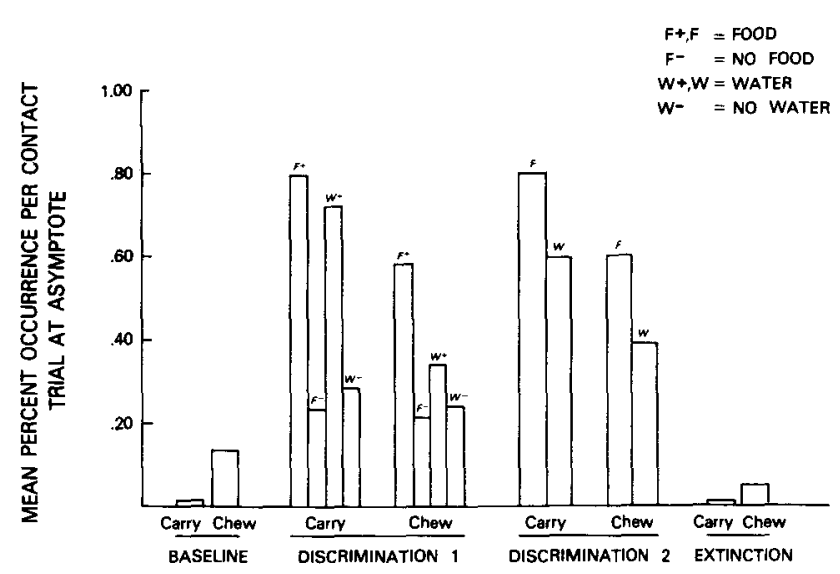

Figure 7. Average percent of contact trials with a carry or chew of bearings predicting food, water, or nothing at asymptote (3-day medians) during baseline, Discrimination 1, Discrimination 2, and extinction (Experiment 3).

Figure 7 also shows no effect of type of reward on the likelihood of a carry or chew, but a strong effect of presence of reward in increasing carry or chew $[F s(1,8)=11.0$ and 3.68 , ps $\langle .01$ and $.10>p\rangle$ .05]. Comparisons with baseline measures revealed an increase in carry or chew only for the rewarded trials. Last, food and water animals did not differ in terms of sniff, but the nonrewarded bearing was sniffed significantly more often than the rewarded bearing $[t(9)=3.75, p<.01]$. Almost no digging occurred under this discrimination.

Discrimination 2. Figure 5 shows that when one bearing predicted food and the other predicted water, the animals contacted on nearly every trial, with no significant difference between the amounts of contact with food and water bearings $[t(9)<1$, p $\rangle$ .10]. Under extinction, contact decreased sharply for both food and water bearings $[\operatorname{ts}(9)=9.97$ and 11.32 , $p<.01]$. Figure 6 shows that animals contacted the food bearing significantly longer than they did the water bearing $[\mathrm{t}(9)=2.58, \mathrm{p}<.01]$, although there was no significant difference in latency to contact $[\mathrm{t}(9)<1, \mathrm{p}\rangle .10]$. However, from the discrimination to the extinction condition there were significant increases in latency to contact both bearings [ts(9) = 4.66 and 4.81 , ps $<.01$ ] and significant decreases in average duration of contact $[\operatorname{ts}(9)=3.92$ and 5.84 , ps $<$.011. Similarly, Figure 7 shows that the animals were more likely to carry and chew the food bearing than the water bearing $[\operatorname{ts}(9)=1.84$ and 1.61 , ps $<$ .05 and $.10>p>.05$ ]. There were also significant decreases in carry and chew for both ball bearings from the discrimination to the extinction conditions $[\operatorname{ts}(9)=6.43,2.39,2.25$, and 2.08 , all ps at least $<.05]$

\section{Discussion}

The average duration of contact and the likelihood of carry and chew were affected by the type of reward and even more clearly by the presence of reward. The animals contacted longer and with greater complexity the ball bearing that predicted reward than the one that predicted no reward, and the ball bearing that predicted food than the one that predicted water. However, the frequency and complexity of contact with bearings that predicted water was much higher than it had been in Experiment 1. Perhaps the less frequent contact by the water animals in Experiment 1 was idiosyncratic, due to the persistence of competing effects of exposure to a larger number of US-alone presentations, or water restriction alone may produce effects different from those produced by the combination of explicit food and water restriction.

It seems more likely, however, that presenting bearings that predict both food and water to the same animal under a common multiple-restriction schedule creates motivational and attentional states (and probably discrimination problems) that favor the expression of more food-related behavior to the water bearing. In contrast with the results of Experiment 1 , the animals in the present experiment did not attempt to dig the bearing out of the entrance hole; instead, they stopped and examined each bearing as it entered the chamber. It appeared that they discriminated between the bearings on the basis of this interaction rather than on the basis of more distant visual inspection or sound. This strategy appeared responsible for the absence of latency differences and probably contributed to the increased similarity in treatment of the food and water bearings.

It is worth noting that Jenkins and Moore (1973) reported somewhat parallel results in the case of pigeons exposed to food and water predicted by different lighted keys. Pigeons exposed only to food or water reward tended to show a greater difference in frequency and form of responding to the predictive keylight than did pigeons exposed to both (although there was at least one water-reward pigeon that showed considerable food-type pecking). Just as in the present case, the locus of change came in more food responding directed to the water key. The pigeons receiving both food and water were judged to engage in food-related pecking to the water key on $30 \%$ of the water trials, but were judged to engage in water-related pecking on only $4 \%$ of the food trials. Whatever the determinants of this phenomenon, it is clear that the rats still reacted differently to bearings related to reward relative to those related to nonreward, and to those related to food relative to those related to water, even when they were under a common deprivation state and were required to make an apparently difficult discrimination. 


\section{GENERAL DISCUSSION}

Taken together, these experiments showed ready conditioning of a complex set of motor behaviors involving contact and various degrees of digging, seizing, carrying, and chewing directed to a rolling ball bearing that predicted either food or water. Conditioning occurred under a modified Pavlovian procedure and under an operant contingency requiring only contact with the bearing. Although the absolute level of conditioning was lower under the Pavlovian procedure, the form of reaction to the bearing did not appear to differ in any important way from the form under the operant procedure. It appears to be an inescapable conclusion that behaviors directed to the bearing were not conditioned individually by accidental juxtaposition of response and reward. Rather, the behaviors appeared to be part of a preorganized pattern related to the moving stimulus of the bearing, the type of reward, and the deprivation state. Elements of this pattern were increased in frequency by a modest degree of association between the bearing and the reward.

The present results generally corroborated previous work on the differences in behavior conditioned with food and water reward. Boakes et al. (1978) found that food-rewarded animals took longer to drop a ball bearing down the exit chute than did water-rewarded animals. Presumably, this delay was based primarily on a more complex and sustained interaction with the bearing by the food animals. Davey and Cleland (1982) found that food-deprived rats tended to sniff, paw, mouth, and bite a lever the insertion of which predicted food, whereas waterdeprived rats showed considerably less interaction with a lever that predicted water. Hull (1977) reported that rats barpressing for food tended to show more long-duration presses than did rats barpressing for water.

Similar differences in reactions were shown in our Experiment 1. Ball bearings predicting food rather than water were engaged more frequently, with shorter latency and longer duration of contact, and tended to elicit more complex motor behaviors, such as dig, carry, and chew. This difference in the effect of food and water reward was modified by prior experience in that the animals tended to persist in previous patterns of reaction despite changes in restriction conditions and reward (Experiment 2). These results are compatible with the results of Boakes et al. (1978) and Hull (1977) in showing persistence of behavior in animals trained under food reward and switched to water reward, but failed to support their report that rats switched from water to food changed their behavior appropriately. However, a comparison of the reactions of animals in the second discrimination condition of Experiment 3 with their reactions in the first discrimination condition suggests that animals can overcome prior conditioning with either food or water alone and respond differentially to bearings predicting food and water in the same situation.

Experiment 3 also showed longer and more complex interactions with bearings that predicted food rather than water. However, reactions to the water bearing were more complex than those found in Experiment 1 . This may have been due to the use of common restriction conditions, the strategies animals adopted to make the discrimination, or the use of intermittent reward. Together, the present experiments show that interaction with a bearing predicting reward is complexly determined by many factors other than simple predictability, including previous experience, the nature of the commodity restricted, the type of reward, and the nature of the experimental task.

The present results, in combination with those of Boakes et al. (1978), Davey and Cleland (1982), Jenkins, Barrera, Ireland, and Woodside (1978), Timberlake (1983, in press), Williams (1981), Woodruff and Starr (1978), and Woodruff and Williams (1976), strongly support the behavior-system view that preorganized behavior patterns related to characteristics of the predictive stimulus and the type of system activated can be conditioned under both Pavlovian and operant procedures. In the present data, when the feeding system was potentiated by restriction of food and use of a food US (reward), behavior directed to moving stimuli that predicted reward involved strong predatory reactions. When the water system was made more salient by restriction of water and use of a water US, behavior involved less frequent, less vigorous, and shorter-duration contact. However, adding both food and water restriction and requiring a discrimination increased frequency and complexity of contact with a water US.

Neither the stimulus-substitution hypothesis nor a simple operant account adequately explain the present data. That animals contacted the bearings without a response requirement and, when required to make a simple contact, did so with such consistent topography and excessive zeal cannot be explained readily by accidental or explicit response contingencies. As for the stimulus-substitution explanation, the animals simply did not treat the bearing as they treated either the food pellets or water obtained in this situation. In the case of a bearing predicting food, an animal would be expected to pick it up in its mouth and chew it. Instead, if often dug the bearing out of its entrance hole, seized it in its paws, stuffed it in its mouth, ran to the other end of the apparatus, and then sat and gnawed it with its incisors, turning it in its paws and dropping and retrieving it. None of these behaviors were ever directed to a food pellet in this situation. In the case of the bearing predicting water, an animal would be expected 
to sniff and lick the bearing. The animals here did come to sniff it frequently, although usually not any more or less than did the food group. Most often, the animals contacted with their paws, but some animals treated the bearing complexly, as though it predicted food. They never licked it in any obvious way.

It has been argued that the stimulus-substitution hypothesis allows a greater range of responding to a predictive stimulus than has been indicated here. By this argument, if an animal acts toward a stimulus predicting reward as it acts toward any example of that type of reward, then stimulus substitution is satisfied. In this broad form, stimulus substitution can include complex predatory and food-handling behaviors, because such behaviors occur toward some examples of food. The behavior of rats toward the bearing that predicted water in the present experiments is still difficult to explain, but in this broad form the predictions of the stimulus substitution hypothesis often converge on those of the behaviorsystem approach.

However, the stimulus-substitution account remains problematic because it lacks the larger adaptive-ecological framework of the behaviorsystem approach. Even a broad stimulus-substitution view obviously fails in the face of appetitive behaviors other than capture and manipulation responses, such as social behaviors (Timberlake, in press, Timberlake \& Grant, 1975) and systematic exploration of the environment (Timberlake 1983). The behavior-system approach calls attention to the circumstances for producing all relevant forms of appetitive behaviors, whether or not they resemble immediate behavior directed to the US. If one wishes to broaden stimulus substitution to add an ecological perspective about what behaviors are available to the animal, then stimulus substitution and the present approach are indistinguishable. I find this stretches the concept of stimulus substitution irremediably beyond its traditional meaning.

But what of the considerable evidence for the more restricted view of stimulus substitution? Jenkins and Moore (1973) found that pigeons pecked a lighted key with water- or food-related pecks, depending on which commodity the key predicted. Davey and Cleland (1982) and Davey, Phillips, and Cleland (1981) showed that food-deprived rats tended to bite a lever that predicted food but to lick a lever that predicted sucrose solution or milk. Breland and Breland (1966) reported that turkeys and porpoises ate small objects that were associated with food. Finally, Premock and Klipec (1981) found that some debeaked pigeons pecked a keylight predicting food and, in doing so, used the same, relatively novel swiping motion they had come to use in obtaining food.

Given such evidence, it might be argued that the most reasonable theoretical account of condi- tioning is a combination of the behavior-system and stimulus-substitution views. However, such a combination approach may not be necessary or helpful at present. The concept of stimulus substitution is often more post hoc than predictive. One examines the conditioned response for behaviors that resemble those elicited by the US and infers substitution on that basis. Furthermore, the stimulus-substitution hypothesis has nothing to say about a role for environmental and predictive stimuli in altering the form of the conditioned response.

Most importantly, the substitution concept is unnecessary because the behavior-system approach predicts the occurrence of substitution-like behaviors when the predictive stimuli resemble the US sufficiently on physical and temporal dimensions to actually elicit aspects of the unconditioned response. Thus, placing a chewable object with an agreeable odor in a dog's mouth just prior to delivering food powder will probably elicit salivation, chewing, and swallowing. Presenting the same stimulus considerably prior to food might still elicit some chewing, but less salivation and swallowing. Presenting only the odor just before delivery of food would probably produce salivation, but less chewing and swallowing. In all cases, the behavior conditioned depends on the interaction of the stimulus conditions with the stimulus sensitivities and response structures provided by the animal.

Jenkins and Moore's (1973) pigeons trained to peck for water may have pecked a key because its shiny illuminated surface was presented in temporal and physical proximity to water. Pecking a key in response to food was related to the key's punctate temporal proximity to food, and to contributing factors such as similar lighting (Steinhauer, 1982) and perhaps the hard surface of the key. The biting and pawing of bars in the studies of Davey and Cleland (1982) and Davey, Phillips, and Cleland (1981) likely resulted from the physical and temporal proximity of the bar to food, and from its sudden insertion into the chamber and subsequent up and down movement as it was manipulated. In the Davey and Cleland (1982) study, licking may have occurred because the rat deposited sucrose on the lever from its mouth and paws. However, the Davey, Phillips, and Cleland (1981) study used a within-subject procedure under which milk was equally likely to be deposited on either bar. It seems probable that physical and temporal proximity played a role, as did the flat surface of the bar. Rats that I have observed to lick bars often twisted their heads and licked the bottom side of the bar, perhaps (and only perhaps) showing a water-gathering strategy used with flat-leafed plants. Temporal and physical proximity and the size and shape of the predictive stimulus probably also determined the ingestive behaviors reported by Breland and Breland (1966). 
The Premock and Klipec (1981) paper is particularly challenging, because it seems to show that the animal can react to the predictive stimulus with an arbitrary US-related behavior acquired as the result of partial debeaking. However, the nature of the behavior described by Premock and Klipec is not entirely foreign to the pigeon's repertoire. Sideways digging and swiping with the beak is often found as a precursor to more direct pecking and serves to remove obstructions such as leaves or litter. A partially debeaked pigeon may very well class the input from its inability to secure grain by direct pecks as the presence of the obstruction. Thus, these data also can be viewed as demonstrating the tendency for animals to show preorganized appetitive patterns to cues that reliably predict food. Although there is no necessity for preorganized responses to rest only on unlearned (innate) responses, much of the evidence points to species-typical behaviors as a key component in the appetitive structure of behavior.

The advantages of the behavior-system approach are, of course, accompanied by some problems. An interesting issue is why the rats in the present experiments showed any complex behaviors at all in response to bearings related to water. Three general possibilities are apparent. Complex manipulation and approach behaviors may be available under water reward because: (1) food-related behaviors are a small but reliable aspect of the water system; (2) the food system is potentiated by the same deprivation manipulations that potentiate the water system; and (3) preorganized perceptual-motor modules, such as predatory behavior to moving stimuli, may occur relatively independenty of particular response systems. These possibilities are not necessarily independent and remain to be evaluated further.

Another important issue is the question of adaptiveness (e.g., Hollis, 1982; Miller \& Balaz, 1981). Presumably, preorganized response structures and stimulus sensitivities evolved to facilitate adjustments to the demands of the environment; thus, in an evolutionary sense, they are adaptive. However, whether they are adaptive in particular experimental circumstances depends upon how closely these circumstances resemble the conditions of the selection environment. In the behavior-system view, phenomena as diverse as egregious misbehavior and an effective simple operant are based on the same underlying organization. The behaviors that occur are determined by the stimulus organization of the environment and the stimulus sensitivities and response organization of the animal (Timberlake et al., 1982).

A final issue is the question of clarity of prediction. The behavior-system approach is closer to a heuristic device than to a final theory. One must be aware of an animal's response structures and stim- ulus sensitivities, and guess how they will interact with experimental conditions. To an extent, experiments have the simultaneous role of testing the behavior-system approach while examining its mapping to behavior and stimulus manipulations. However, these same objections apply in at least the initial studies of all conditioning hypotheses. As indicated above, behavioral definitions of stimulus substitution are frequently hazy; they are worked out after results are obtained. Similarly, the identification of reinforcers is made after the fact. The strength of the behavior-system approach is the provision of an analytic framework that calls attention to behaviors and stimulus sensitivities that can be examined beforehand, independently of the conditioning situation.

The grounding of the behavior-system approach in species-typical response structures and stimulus sensitivities is sometimes viewed as a weakness. The tradition in animal learning has been to couch theories in species-independent terms, relegating consideration of species differences to the design of the apparatus and procedures (Timberlake, 1983). This procedure has been presumed to be essential to the development of a general theory of learning that applies to all species. However, the behavior-system approach shows that a conditioning approach can include species-typical behaviors and still be general; it simply attempts to elevate the contribution of the species from the status of laboratory lore to explicit theory. In the long run, I think this will provide the most satisfactory general account of learning.

In sum, the behavior-system approach accounts for the conditioning of behaviors ranging from appetitive components well removed from the terminal event in a system to aspects of the terminal response itself. The outcome depends on the nature and timing of the predictive stimuli and the stimulus and motivational support in the environment. Unfortunately, the traditional concentration on the terminal response has tended to bind animal learning to a single behavioral focus and time frame for studying conditioning. Because autonomic responses such as salivation are controlled only by predictive stimuli that immediately precede delivery of the US, much previous research has concluded that learning about signals must be restricted to a very short temporal interval. However, these conclusions were based on experiments using an extremely restricted set of behaviors as the index of conditioning. As every experimenter knows, although the animal may not salivate on its way to the experimental room, it does show considerable anticipatory locomotor and searching behavior long before the experimental room, much less the apparatus or the specific predictive stimuli used in the experiment, is in sight. If we wish to develop a general ecologically valid theory of learning, we need 
to consider the more complete set of appetitive behaviors and physical and temporal sensitivities characteristic of the animal.

\section{REFERENCE NOTE}

1. Timberlake, W. Personal observation, 1977.

\section{REFERENCES}

Adams, C., \& Dickinson, A. Actions and habits: Variations in associative representations during instrumental learning. In N. E Spear \& R. R. Miller (Eds.), Information processing in animals: Memory mechanisms. Hillsdale, N.J: Erlbaum, 1981.

Bonkes, R. A. Performance on learning to associate a stimulus with positive reinforcement. In H. Davis \& H. M. B. Hurwitz (Eds.), Operant-Pavlovian interactions. Hillsdale, N.J: Erlbaum, 1977.

Bonkes, R. A., \& Jefrery, G. Automodellaggio e malcomportamento. Richerche di Psicologia, 1979, 10, 53-68.

Bonkes, R. A., Poli, M., Lockwood, M. J., \& Goodall, G. A study of misbehavior: Token reinforcement in the rat. Journal of the Experimental Analysis of Behavior, 1978, 29, 115-134.

Bolles, R. C. Readiness to eat: Effects of age, sex, and weight loss. Journal of Comparative and Physiological Psychology, $1965,60,88-92$.

Bolles, R. C. Theory of motivation (2nd ed.). New York: Harper \& Row, 1975.

Breland, K., \& Brelanồ, M. Animal behavior. New York: Macmillan, 1966.

Davey, G. C. L., \& Cueland, G. G. Topography of signal-centered behaviour in the rat: Effects of deprivation state and reinforcer type. Journal of the Experimental Analysis of Behavior, 1982, 38, 291-314.

Davey, G. C. L., Phillips, S., \& Cleland, G. G. The topography of signal-centered behaviour in the rat: The effects of solid and liquid food reinforcers. Behaviour Analysis Letters, 1981, 1, 331-337.

Hollis, K. L. Pavlovian conditioning of signal-centered action patterns in autonomic behavior: $A$ biological analysis of function. In J. S. Rosenblatt, R. A. Hinde, C. Beer, M.-C. Busnel (Eds.), Advances in the study of behavior (Vol. 12). New York: Academic Press, 1982.

HuL.L, J. H. Instrumental response topographies of rats. Animal Learning \& Behavior, 1977, 5, 207-212.

Jenkins, H. M., Barrera, C., Ireland, C., \& Woodgide, B. Signal-centered action patterns of dogs in appetitive classical conditioning. Learning and Motivation, 1978, 9, 272-296.

Jenkins, H. M., \& Moore, B. R. The form of the autoshaped response with food or water reinforcers. Journal of the Experimental A nalysis of Behavior, 1973, 20, 163-182.

KarLI, P. The Norway rat's killing response to the white mouse: An experimental analysis. Behaviour, 1956, 10, 81-103.

Mackintosh, N. M. The psychology of animal learning. New York: Academic Press, 1974.
Miller, R. R., \& Balaz, M. A. Differences in adaptiveness between classically conditioning responses and instrumentally acquired responses. In N. E. Spear \& R. R. Miller (Eds.), Information processing in animals: Memory mechanisms. Hillsdale, N.J: Erlbaum, 1981.

Moore, B. R. The role of directed Pavlovian reactions in simple instrumental learning in the pigeon. In R. A. Hinde \& J. Stevenson-Hinde (Eds.), Constraints on learning: Limitations and predispositions. London: Academic Press, 1973.

Pavlov, I. P. [Conditioned reflexes] (G. V. Anrep, trans.). London: Oxford University Press, 1927.

Premock, M., \& KLIPEc, W. D. The effects of modifying consummatory behavior on the topography of an autoshaped pecking response in pigeons. Journal of the Experimental Analysis of Behavior, 1981, 36, 277-284.

Reberg, D., Innis, N. K., Mann, B., \& Eizenga, C. "Superstitious" behavior resulting from periodic response-independent presentations of food or water. Animal Behaviour, 1978, 26, 506-519.

Rege ro, D., Mann, B., \& Innis, N. K. Superstitious behaviour for food and water in the rat. Physiology \& Behavior, 1977, $19,803-806$.

Steinhauer, G. D. Acquisition and maintenance of autoshaped key pecking as a function of food stimulus and key stimulus similarity. Journal of the Experimental Analysis of Behavior, 1982, 38, 281-291.

Timberlake, W. Appetitive structure and straight alley running. In R. Melgren (Ed.), Animal cognition and behaviour. Amsterdam: North-Holland, 1983.

Timgerlake, $W$. The functional organization of appetitive behavior: Behavior systems and learning. In M. D. Zeiler \& P. Harzem (Eds.), Advances in analysis of behavior: (Vol. 3) Biological factors in learning. Chichester, England: Wiley, in press.

Timberlake, W., \& Grant, D. L. Autoshaping in rats to the presentation of another rat predicting food. Science, 1975, 190, 690-692.

Timberlake, W., Wahl, G., \& King, D. Stimulus and response contingencies in the misbehavior of rats. Journal of Experimental Psychology: Animal Behavior Processes, 1982, 8, 62-85.

Williams, D. R. Biconditional behavior: Conditioning without constraint. In C. M. Locurto, H. S. Terrace, \& J. Gibbon (Eds.) Autoshaping and conditioning theory. New York: Academic Press, 1981.

Woodruff, G., \& Stakr, M. D. Autoshaping of initial feeding and drinking reactions in newly hatched chicks. Animal Learning \& Behavior, 1978, 6, 265-272.

WoODnuFF, G., \& Williams, D. R. The associative relation underlying autoshaping in the pigeon. Journal of the Experimental Analysis of Behavior, 1976, 26, 1-13.

(Manuscript received December 14, 1982; revision accepted for publication May 11, 1983.) 